\title{
JUPITER strikes earth
}

\author{
Valentin Fuster and Sameer Bansilal
}

The field of cardiovascular primary prevention has truly taken on global proportions and grows everyday with mounting interest from patients, physicians, insurers, national and international health agencies, and governments worldwide. This enthusiasm for research on primary prevention is encouraging; however, studies should be designed and interpreted with their broad context-including the economics of healthcare-in mind. JUPITER (Ridker PM et al. [2008] N Engl J Med 359: 2195-2207) serves as a good example of the new wave of cardiovascular prevention trials from which many lessons can be learnt.

JUPITER was a large, multinational, 4-year, double-blind, placebo-controlled, randomized clinical trial that included 17,802 apparently healthy men and women assigned to rosuvastatin $20 \mathrm{mg}$ or placebo. The study was designed to assess whether statin therapy should be given to individuals with normal levels of LDL cholesterol (<130 mg/dl), but elevated levels of $\mathrm{C}$-reactive protein (CRP; $>2.0$ $\mathrm{mg} / \mathrm{l})$. In patients assigned to rosuvastatin, LDL-cholesterol and CRP levels were halved, and triglyceride levels were reduced by $17 \%$ after 12 months of treatment. After 1.9 years of follow-up, treatment with rosuvastatin significantly reduced the primary composite end point by $44 \%$, as well as nearly all of the individual end points - including a 55\% reduction in nonfatal myocardial infarction, a $48 \%$ reduction in the risk of nonfatal stroke, and a $47 \%$ reduction in the risk of hard cardiac events (a composite of myocardial infarction, stroke, and death from cardiovascular causes) - compared with placebo. The trial was halted by the Data and Safety Monitoring Board.

Although JUPITER clearly establishes the efficacy of rosuvastatin for the reduction of cardiovascular events, it leaves us with a host of unanswered clinical and mechanistic questions.
JUPITER ....

serves as a

good example

of the new

wave of

cardiovascular

prevention

trials from

which many

lessons can be

learnt.

$V$ Fuster is the Editorin-Chief of Nature

Clinical Practice

Cardiovascular

Medicine, and

Director of Mount

Sinai Heart, the Zena

and Michael A Wiener

Cardiovascular

Institute, and the

Marie-José and Henry

$R$ Kravis Center

for Cardiovascular

Health, Mount Sinai

School of Medicine,

New York. S Bansilal

is a Cardiology Fellow

at the Mount Sinai

Cardiovascular

Institute, New York,

NY, USA.

\section{Competing interests}

The authors declared no

competing interests.

www.nature.com/clinicalpractice doi:10.1038/ncpcardio1454
The long-term safety of aggressive lipid lowering, and the questionable increase in the incidence of physician-reported diabetes in the rosuvastatin arm (3\% versus $2.4 \%$, number needed to harm $[\mathrm{NNH}]=166)$, are left in doubt. Furthermore, although the study demonstrated high relative-risk reductions, the overall incidence of the composite primary end point was only $2.8 \%$ in the placebo arm, with an absolute risk reduction of $1.2 \%(\mathrm{NNH}=83)$, and the incidence of hard cardiovascular end points was only $1.8 \%$, with an absolute risk reduction of $0.9 \%(\mathrm{NNH}=120)$.

Finally, almost 90,000 individuals were screened to establish the study population that the investigators perhaps inaccurately labeled as low-risk, since almost half of the participants had a Framingham risk score of $10 \%$ or higher, and $41 \%$ of participants had the metabolic syndrome. To define the utility of high-sensitivity (hs) CRP measurement as a strategy for identifying individuals for treatment would require a study where traditional risk factors guide management in one arm, and hs-CRP levels would guide management in the other. Issues that impact on the cost to the health-care system -including requirement for large screening programs, relatively low yields, expensive drugs, etc. - are very important considerations when evaluating the impact that any trial should have on treatment strategies.

JUPITER falls into the category of lipidlowering primary prevention studies, and establishes the paradigm of 'the lower the LDL-cholesterol level the better', a maxim that already existed for secondary cardiovascular prevention. Nevertheless, JUPITER is a true landmark in preventive cardiology, not only for its findings, which will certainly affect primary prevention lipid guidelines, but even more so for the challenges it raises to our current strategies for risk assessment. 


\section{EDITORIAL}

www.nature.com/clinicalpractice/cardio

\section{CORRECTION}

\section{JUPITER strikes earth}

Valentin Fuster and Sameer Bansilal

Original article Fuster, V. \& Bansilal, S. JUPITER strikes earth. Nat. Clin. Pract. Cardiovasc. Med. 6, 159 (2009).

The third sentence in paragraph 3 should have read "Furthermore, although the study demonstrated high relative-risk reductions, the overall incidence of the composite primary end point was only $2.8 \%$ in the placebo arm, with an absolute risk reduction of $1.2 \%$ (number needed to treat $=83$ ), and the incidence of hard cardiovascular end points was only $1.8 \%$, with an absolute risk reduction of $0.9 \%$ (number needed to treat $=120$ )" rather than number needed to harm, as was indicated.

doi:10.1038/nrcardio.2009.54 